\title{
Isoliquiritigenin, an Orally Available Natural FLT3 Inhibitor from Licorice, Exhibits Selective Anti-Acute Myeloid Leukemia Efficacy In Vitro and In Vivo
}

\author{
Zhi-Xing Cao, ${ }^{1}$ Yi Wen, ${ }^{1}$ Jun-Lin He, Shen-Zhen Huang, Fei Gao, Chuan-Jie Guo, \\ Qing-Qing Liu, Shu-Wen Zheng, Dao-Yin Gong, Yu-Zhi Li, Ruo-Qi Zhang, Jian-Ping Chen, \\ and Cheng Peng
}

Pharmacy College, Chengdu University of Traditional Chinese Medicine, Ministry of Education Key Laboratory of Standardization of Chinese Herbal Medicine, Key Laboratory of Systematic Research, Development and Utilization of Chinese Medicine Resources in Sichuan Province-Key Laboratory Breeding Base of Co-founded by Sichuan Province and MOST, Chengdu, China (Z.-X.C., J.-L.H., C.-J.G., S.-W.Z., D.-Y.G., Y.-Z.L., R.-Q.Z., J.-P.C., C.P.);School of Chinese Medicine, University of Hong Kong, Hong Kong, China (Y.W., F.G., Q.-Q.L., J.-P.C.); College, Shenzhen Institute of Research and Innovation, University of Hong Kong, Shenzhen, China (Y.W., F.G., Q.-Q.L., J.-P.C.); and State Key Laboratory of Biotherapy and Cancer Center, West China Hospital, Sichuan University, Chengdu, China (S.-Z.H.)

Received February 19, 2019; accepted August 20, 2019

\section{ABSTRACT}

Licorice is a medicinal herb widely used to treat inflammationrelated diseases in China. Isoliquiritigenin (ISL) is an important constituent of licorice and possesses multiple bioactivities. In this study, we examined the selective anti-AML (acute myeloid leukemia) property of ISL via targeting FMS-like tyrosine kinase3 (FLT3), a certified valid target for treating AML. In vitro, ISL potently inhibited FLT3 kinase, with an $\mathrm{IC}_{50}$ value of $115.1 \pm 4.2$ $\mathrm{nM}$, and selectively inhibited the proliferation of FLT3-internal tandem duplication (FLT3-ITD) or FLT3-ITD/F691L mutant AML cells. Moreover, it showed very weak activity toward other tested cell lines or kinases. Western blot immunoassay revealed that ISL significantly inhibited the activation of FLT3/Erk1/2/signal transducer and activator of transcription 5 (STAT5) signal in

\section{Introduction}

Acute myeloid leukemia (AML) is the most frequently diagnosed type of aggressive hematologic malignancy in adults, with more than 20,000 newly diagnosed patients in America every year (Siegel et al., 2015). Although the mortality has substantially declined over the past two decades with the implementation of therapeutic modalities of standard

This study was supported by the National Natural Science Foundation of China [Grants 81873073, 81573663, 81503346, and 81630101], International Science Foundation of Guganxi [Grant AB16450008], National Major Scientific and Technological Special Project of China [Grant 2017ZX09201001-008], Youth Scientific Research Fund of CDUTCM [Grants ZRQN1764 and ZRQN1770], and Sichuan Science and Technology Program [Grant 2018SZDZX0017].

The authors declare no conflicts of interests.

${ }^{1}$ Z.-X.C., and Y.W. contributed equally to this work.

https://doi.org/10.1124/mol.119.116129.
AML cells. Meanwhile, a molecular docking study indicated that ISL could stably form aromatic interactions and hydrogen bonds within the kinase domain of FLT3. In vivo, oral administration of ISL significantly inhibited the MV4-11 flank tumor growth and prolonged survival in the bone marrow transplant model via decreasing the expression of Ki67 and inducing apoptosis. Taken together, the present study identified a novel function of ISL as a selective FLT3 inhibitor. ISL could also be a potential natural bioactive compound for treating AML with FLT3-ITD or FLT3-ITD/F691L mutations. Thus, ISL and licorice might possess potential therapeutic effects for treating AML, providing a new strategy for antiAML.

chemotherapy, chemotherapy resistance and intolerance are inevitable (Döhner et al., 2017; Podoltsev et al., 2017). Consequently, the identification and development of safer and more effective anti-AML agents are highly desirable.

The high relapse rates of AML may be attributed partially to genetic and epigenetic heterogeneity and chemoresistanceenabling mutations (Papaemmanuil et al., 2016; Tsai et al., 2016). Moreover, nearly $30 \%$ of AML patients carry activating mutations in the FMS-like tyrosine kinase-3 (FLT3) gene, which encodes a class III receptor tyrosine kinase and regulates the normal hematopoiesis (Gilliland and Griffin, 2002; Prada-Arismendy et al., 2017). The accumulated evidence over the past years indicated that internal tandem duplication mutations in the juxtamembrane domain of FLT3 kinase (FLT3-ITD) are the most prevalent mutations that lead to uncontrolled cellular proliferation and survival

ABBREVIATIONS: AML, acute myeloid leukemia; DS, Discovery Studio; FITC, fluorescein isothiocyanate; FLT3, FMS-like tyrosine kinase-3; GOLD, Genetic Optimization for Ligand Docking; HTRF, homogeneous time-resolved fluorescence; IL-3, interleukin 3; ISL, isoliquiritigenin; ITD, internal tandem duplication; MST, mean survival time; MTT, 3-(4,5-dimethylthiazol-2-yl)-2,5-diphenyltetrazolium; PI, propidium iodide; STAT5, signal transducer and activator of transcription 5; TKD, tyrosine kinase domain; TUNEL, terminal deoxynucleotidyl transferase-mediated dUTPbiotin nick end-labeling. 
characterized by constitutive ligand-independent activation of FLT3 (Kiyoi et al., 2002; Levis et al., 2018). Several studies have demonstrated that patients with high FLT3ITD allelic ratios exhibit a high relapse rate, poor prognosis, and worse survival rate (Döhner et al., 2017; El Fakih et al., 2018). Recently, small-molecule FLT3 inhibitor midostaurin has been demonstrated to effectively inhibit the growth of FLT3-ITD-positive AML cells in animal and human, and has been approved by the US Food and Drug Administration for treating AML (Stone et al., 2017). Moreover, a number of small-molecule FLT3 inhibitors, including quizartinib (Cortes et al., 2018), sunitinib (Fiedler et al., 2015), ponatinib (Shah et al., 2013), MLN-518 (Odia et al., 2016), and gilteritinib (Perl et al., 2017), are being studied in clinical trials and have yielded favorable results against AML FLT3-ITD mutant isoforms. Although these FLT3 inhibitors exhibit potent anti-AML activity in clinical trials, drug resistance and relapse often occur. Recently, point mutational analysis showed that F691L mutation in the tyrosine kinase domain (TKD) of FLT3-ITD confers acquired resistance to these drugs (Albers et al., 2013; Xu et al., 2017). Thus, acquired resistance is still a challenge in the drug development of FLT3-targeted therapy.

Isoliquiritigenin (ISL), a natural flavonoid mainly extracted from licorice root, is one of the most widely used traditional Chinese medicines in China, and is often used in food flavoring in Western countries. Previous studies have demonstrated that ISL possesses various pharmacological actions, including anti-inflammatory (Zeng et al., 2017), antiplatelet aggregation (Tawata et al., 1992), spasmogenic (Liu et al., 2008), antimicrobial (Boyapelly et al., 2017), vasorelaxant (Yu and Kuo, 1995), and estrogenic effects (Kundu et al., 2018). Moreover, ISL has been proven to possess significant inhibitory activities against liver (Hsu et al., 2005), colon (Wu et al., 2016), prostate (Zhang et al., 2018a), and cervical cancer cells via inducing apoptosis or autophagy, cell cycle arrest, or inhibiting migration and triggering oxidative stress (Peng et al., 2015; Tsai et al., 2015). Previously, our group demonstrated that ISL exhibited a significant inhibitory effect on breast cancer through suppressing angiogenesis, repressing cancer stem cells, regulating autophagy, and modulating miR-374a (Wang et al., 2013, 2014, 2015; Peng et al., 2017). These results suggested that ISL is a multifunctional natural bioactive compound with potent anticancer activities. However, there is little literature on the role and targets of ISL in human AML.

The results of our present study demonstrated that ISL could selectively inhibit the activation of the FLT3 kinase and the viability of FLT3-ITD mutant AML cell lines with negligible activity in other types of tumor cells and normal cells in vitro. Furthermore, ISL could significantly inhibit tumor growth of MV4-11 in a xenograft tumor model and bone marrow model with good tolerance. Taken together, the present study identified a novel function of ISL as a selective FLT3 inhibitor as well as a potential natural bioactive compound for treating AML with FLT3-ITD mutations.

\section{Materials and Methods}

Materials. For the present study, 3-(4,5-dimethylthiazol-2-yl)-2,5diphenyltetrazolium (MTT), dimethylsulfoxide (DMSO), and polyethylene glycol 400 were procured from Sigma Chemical Co. (St. Louis, MO). Rabbit anti-phosphorylated-FLT3 (Tyr969, \#3463), rabbit anti-FLT3 (8F2, \#3462), rabbit anti-phosphorylated-STAT5 (Tyr105, \#3827), rabbit anti-STAT5 (\#9363), rabbit anti-phosphorylatedErk1/2 (Thr202/Tyr204, \#4370), rabbit anti-Erk1/2 (\#4695), mouse anti$\beta$-actin (\#3700) was purchased from Cell Signaling Technology (Beverly, MA), whereas rabbit anti-caspase-3 (ab184787) was purchased from Abcam (Cambridge, MA). Isoliquiritigenin, with a purity of more than 97\%, was obtained from Alfa Aesar (Haverhill, MA). Sunitinib was acquired from Nanjing Chemlin Chemical Industry Co. Ltd. (Nanjing, Jiangsu). All of the chemicals used in this study were of analytical grade.

Cell Culture. Unless otherwise stated, the cell lines were obtained from the American Type Culture Collection (Manassas, VA). All cells, except for MV4-11 cells, were grown in RPMI 1640 or Dulbecco's modified Eagle's medium culture medium supplemented with $10 \%$ fetal bovine serum (v/v), $100 \mathrm{U} / \mathrm{ml}$ penicillin, and $100 \mathrm{U} / \mathrm{ml}$ streptomycin. MV4-11 cells were cultured in the Iscove's modified Dulbecco's medium culture medium containing $10 \%$ fetal bovine serum $(\mathrm{v} / \mathrm{v})$ and antibiotics. All cultures were maintained at $37^{\circ} \mathrm{C}$ and $5 \% \mathrm{CO}_{2}$.

Cell Viability Assays. Cell viability was measured with the colorimetric MTT metabolic activity assay. In brief, the leukemic cells were seeded in a 96 -well plate at a density of $1-4 \times 10^{4}$ cells per well for 24 hours, and an equal volume of medium containing an increasing concentration of inhibitors was added to each well. The other cell lines were seeded in 96 -well plates at a density of $2-5 \times 10^{3}$ cells/well for 24 hours, and then the medium was replaced with the medium containing serial dilutions of inhibitors. After 72 hours of further incubation, $20 \mu \mathrm{l}$ of $5 \mathrm{mg} / \mathrm{ml}$ MTT reagent was added into each well followed by $2-4$ hours of incubation. Subsequently, $50 \mu \mathrm{l}$ of $20 \%$ acidified SDS per well was added to lyse the cells. The absorbance values of the dissolved cells were measured at $570 \mathrm{~nm}$ with a SpectraMAX M5 microplate spectrophotometer (Molecular Devices). All experiments were performed in triplicate. Cell viability was calculated as the percentage of viable cells relative to the control cells treated with DMSO $(0.1 \%)$.

In Vitro Kinase Assay. The recombinant human protein FLT3 was obtained from Eurofins. The inhibition of the FLT3 kinase activity was measured with homogeneous time-resolved fluorescence (HTRF) assays. Optimal enzyme and substrate concentrations were determined with the HTRF KinEASE kit (Cisbio) according to the manufacturer's instructions. In brief, the FLT3 enzymes were mixed with peptide substrate in a kinase reaction buffer system containing $50 \mathrm{mM}$ (pH 7.0) HEPES, $0.02 \% \mathrm{NaN}_{3}, 0.1 \mathrm{mM}$ sodium orthovanadate, $5 \mathrm{mM} \mathrm{MgCl}_{2}, 0.01 \%$ (w/v) bovine serum albumin, $1 \mu \mathrm{M}$ Tyrosine kinase substrate-biotin, and $2 \mu \mathrm{M}$ ATP. The final concentration of FLT3 in the system was $6 \mathrm{nM}$. The reaction was quenched by addition of development reagent, followed by incubation for an additional hour prior to fluorescence measurements on EnVision Multilabel Reader (PerkinElmer). Protein kinase profiling of ISL was also evaluated with HTRF assay in a panel of five human protein kinases. The percentage inhibition was calculated relative to an enzyme control without inhibitor. $\mathrm{IC}_{50}$ values were calculated by four-parameter nonlinear regression analysis of the resulting concentration-response curves using GraphPad PRISM (GraphPad Software).

Enzyme Kinetic Experiments. To investigate whether the ATP binding site is the binding site of ISL with FLT3, the enzyme kinetic experiments were performed with an increasing concentration of $\operatorname{ATP}(0.16-200 \mu \mathrm{M})$ in the kinase assay mentioned earlier.

Cell Cycle Analysis. For cell cycle analysis, MV4-11 cells were seeded into six-well plates at a cell density of $2 \times 10^{5} /$ well. After 30 minutes, cells were treated with different concentrations of ISL $(0.625,2.5$, and $10 \mu \mathrm{M})$ for 20 hours. The cells were harvested and washed in cold PBS. Then, the cells were fixed with $95 \%$ ice-cold ethanol and treated with RNase A $(100 \mu \mathrm{g} / \mathrm{ml})$ and stained with propidium iodide (PI; $10 \mu \mathrm{g} / \mathrm{ml}$ ). Cell cycle distribution was analyzed by flow cytometry (BD Biosciences).

Cell Apoptosis. For cell apoptosis analysis, an annexin V-fluorescein isothiocyanate (FITC) apoptosis detection kit (KeyGen BioTECH) was 
used. In brief, the MV4-11 cells were seeded into six-well plates at a cell density of $1 \times 10^{6} /$ well. After 30 minutes, cells were treated with different concentrations of ISL $(2.5,10$, and $40 \mu \mathrm{M})$ for 30 hours. The cells were washed twice with cold PBS and then incubated with annexin V-FITC/PI at room temperature for 5 minutes in the dark. The fluorescence of the cells was detected by flow cytometry by using an FITC signal detector and a PI signal detector (BD Biosciences).

The level of apoptosis was also analyzed by detecting the level of cleaved caspase-3. In brief, MV4-11 cells were treated with ISL at indicated concentrations for 30 hours, and the cells were subsequently lysed with radioimmunoprecipitation assay buffer $[10 \mathrm{mM}$ Tris- $\mathrm{HCl}$ (pH 7.8), $1 \%$ NP40, $0.15 \mathrm{M} \mathrm{NaCl}, 1 \mathrm{mM}$ EDTA, $10 \mu \mathrm{M}$ aprotinin, $1 \mathrm{mM}$ $\mathrm{NaF}$, and $\left.1 \mathrm{mM} \mathrm{Na} \mathrm{VO}_{4}\right]$. The extracted samples were subjected to western analysis as described in the next section using an anticaspase-3 antibody and glyceraldehyde-3-phosphate dehydrogenase as the control.

Western Blot Analysis. Cells (MV4-11) were treated with a series of concentrations of ISL and harvested after 20 hours, then washed with precooled PBS. Total protein was extracted in ice-cold radioimmunoprecipitation assay lysis [10 mM Tris- $\mathrm{HCl}$ (pH 7.8), $1 \% \mathrm{NP} 40$, $0.15 \mathrm{M} \mathrm{NaCl}, 1 \mathrm{mM}$ EDTA, $10 \mu \mathrm{M}$ aprotinin, $1 \mathrm{mM} \mathrm{NaF}$, and $1 \mathrm{mM}$ $\mathrm{Na}_{3} \mathrm{VO}_{4}$ ]. Protein concentration was determined using a BCA (Beyotime Biotechnology, Shanghai) protein quantification assay kit. For western blot assay, the proteins were resolved with 10\%-15\% SDSPAGE gel electrophoresis followed by transfer to polyvinylidene fluoride membranes (Millipore, Billerica, MA) and incubated overnight with the appropriate antibodies. Primary antibodies against FLT3/phosphorylated-FLT3, STAT5/phosphorylated-STAT5, Erk1/ $2 / \mathrm{p}$-Erk $1 / 2$, and $\beta$-actin were used to probe with proteins on the membrane at $4^{\circ} \mathrm{C}$ overnight. Subsequently, the membranes were washed three times with Tris-buffered saline with Tween and incubated with horseradish peroxidase-conjugated secondary antibody for 2 hours at room temperature. Specific antibody binding was detected by an electrochemiluminescence kit on Kodak X-ray films.

Computational Docking Studies. Comparative receptor structure models for FLT3/F691L, a point mutation of FLT3, were generated with the "Build Mutants" module of the Discovery Studio (DS) 3.1 (Accelrys, Inc.) program package. Molecular docking studies were performed using the cocrystal structures of FLT3 with the AC220 (quizartinib, a type II kinase inhibitor) as templates to build the receptor structure of point mutations. The best set of parameters in the DS 3.1 program identified during parameter optimization comprises the following settings. Parameter value of mutant was set as PHE691LEU. The input protein molecule was set to 4XUF, a number of models were set to 5, optimization level was set to high, and other parameters were set as "Build Mutants Default."

Genetic Optimization for Ligand Docking (GOLD) can dock flexible ligands into a protein active site based on the genetic algorithm. Thus, GOLD was used for docking studies, and all of the GOLD protocols were executed in the program package of DS 3.1. The docking calculations were set to add hydrogen in the homology modeling of 4XUF.M0005 and define the receptor for 4XUF.M0005. Then, the binding site containing the ligand of AC220 was defined as an active sphere whose radius was set to $9 \AA$ in this crystal structure. The default calculation mode which provided the best-docked results was selected for calculations.

Effect of ISL on AML Cell Growth In Vivo. Seven- to 8-week-old female NOD-SCID mice were obtained and housed in a sterile environment and fed a standard diet ad libitum. The animals were cared for in accordance with the Guide for the Care and Use of Laboratory Animals of the National Institutes of Health. To establish the AML xenograft model, single-cell suspensions of MV4-11 $\left(6 \times 10^{7}\right.$ cells $/ \mathrm{ml}$ ) in serum-free medium were injected into the hind flank at $100 \mu \mathrm{l} /$ site. When the tumors reached a size of $100-200 \mathrm{~mm}^{3}$, the mice bearing tumors were equally divided into different groups (six mice in each group) and received an oral dose of $\operatorname{ISL}(25,50$, and $100 \mathrm{mg} / \mathrm{kg}$ per day p.o., respectively) or vehicle ( $5 \% \mathrm{DMSO}+20 \%$ polyethylene glycol $400+75 \%$ normal saline) for 18 days. Positive control group mice were injected with sunitinib (10 mg/kg per day by mouth). Tumor growth and body weight were measured every 3 days with a Vernier caliper during treatment in vivo, and the tumor volume was calculated using the following formula: volume $\left(\mathrm{mm}^{3}\right)=\mathrm{a} \times \mathrm{b}^{2} / 2$ [a, longest diameter (length); $b$, shortest diameter (width)]. The mice were closely monitored and weighed. At the end of the experiment, animals were euthanized, and the tumors were harvested and subjected to histologic examination.

Effect of ISL in the Bone Marrow Engraftment Model. NODSCID mice were pretreated with cyclophosphamide at a dose of $150 \mathrm{mg} / \mathrm{kg}$ i.p. once a day for 2 days. After 24 hours, each mouse was intravenously injected with $5 \times 10^{6}$ MV4-11 cells via the tail vein. After 20 days of inoculation, ISL or vehicle was administered orally once a day for 30 days. Positive control group mice were injected with sunitinib ( $10 \mathrm{mg} / \mathrm{kg}$ per day). Survival was determined by observation of the animals without hind-limb paralysis, and those that were moribund were counted as dead.

Immunohistochemistry. Tumor samples obtained from in vivo studies were rinsed, fixed with $10 \%$ paraformaldehyde/PBS, and embedded in paraffin. The femoral bones of bone marrow-engrafted mice were fixed, decalcified, and embedded in paraffin. For immunohistochemical analysis, paraffin-embedded tumors were sectioned $(4-8 \mu \mathrm{m})$ and subjected to immunostaining with Ki67. Furthermore, apoptosis was detected by terminal deoxynucleotidyl transferase-mediated dUTP-biotin nick end-labeling (TUNEL) assay as described by the manufacturer. In brief, deparaffinized sections were permeabilized with $0.1 \%$ Triton X-100 solution and then incubated with $50 \mu \mathrm{l}$ of TUNEL reaction mixture at $37^{\circ} \mathrm{C}$ for 60 minutes. After rinsing thrice with PBS, $50 \mu$ l of converterPeroxidase was added, and the tissue cells were incubated for 30 minutes at $37^{\circ} \mathrm{C}$. Subsequently, the sections were stained using diaminaobenzidine substrate and counterstained with hematoxylin. Apoptotic cells were then visualized with a Leica digital camera attached to a light microscope.

Statistical Analysis. All in vitro experiments were performed in triplicate and replicated more than three times. The data were expressed as means \pm S.D. Student's $t$ test was used to compare the differences between two groups; for multiple comparisons, a post hoc test using Dunnett's method was used to assess the differences between the treated groups and control group; to assess the survival rate of mice, a Kaplan-Meier curve was performed. All statistical analyses were conducted using GraphPad Prism (version 5.01). The value $P<0.05$ was considered to be statistically significant.

\section{Results}

In Vitro Growth Inhibitory Activity of ISL against Leukemia and Other Cell Lines. The in vitro growth inhibitory activities of ISL were evaluated against a panel of tumor cell lines, including leukemia and solid tumor cell lines. As presented in Table 1, ISL selectively inhibited the viability of FLT3-ITD mutant AML cell lines, including

\section{TABLE 1}

Antiproliferative profile of ISL on various cell lines $n=3$ for each experiment

\begin{tabular}{llc}
\hline Cell Lines & \multicolumn{1}{c}{ Tumor Types } & $\mathrm{IC}_{50}$ \\
\hline & & $\mu M$ \\
MV4-11 & Human acute myeloid leukemia & $3.2 \pm 1.2$ \\
MOLM-13 & Human acute myeloid leukemia & $4.9 \pm 2.1$ \\
OCI-LY10 & Human B cell lymphoma & $20.1 \pm 6.7$ \\
A549 & Human lung cancer & $>50$ \\
HGC-27 & Human gastric cancer & $>50$ \\
HepG2 & Human hepatocellular carcinoma & $>50$ \\
HCT116 & Human colorectal cancer & $>50$ \\
MCF-10A & Human mammary epithelial cell & $>50$ \\
Ba/F3 & Murine pro-B cell line & $>50$ \\
\hline
\end{tabular}


MV4-11 and MOLM-13, in a dose-dependent manner, with $\mathrm{IC}_{50}$ values of $3.2 \pm 1.2 \mu \mathrm{M}(0.85 \pm 0.32 \mu \mathrm{g} / \mathrm{ml})$ and $4.9 \pm$ $2.1 \mu \mathrm{M}$, respectively. However, a relatively weak inhibitory activity was observed in human $\mathrm{B}$ cell lymphoma cell lines (OCI-LY10; $\left.\mathrm{IC}_{50}=20.1 \pm 6.7 \mu \mathrm{M}\right)$. Negligible activity was detected against the normal human epithelial mammary cell line MCF-10A and other human common solid tumor cell lines (Table 1). These results indicated that AML cells with FLT3-ITD mutant were extremely sensitive to ISL compared to other cell lines tested.
A<smiles>O=C(/C=C/c1ccc(O)cc1)c1ccc(O)cc1O</smiles>

C

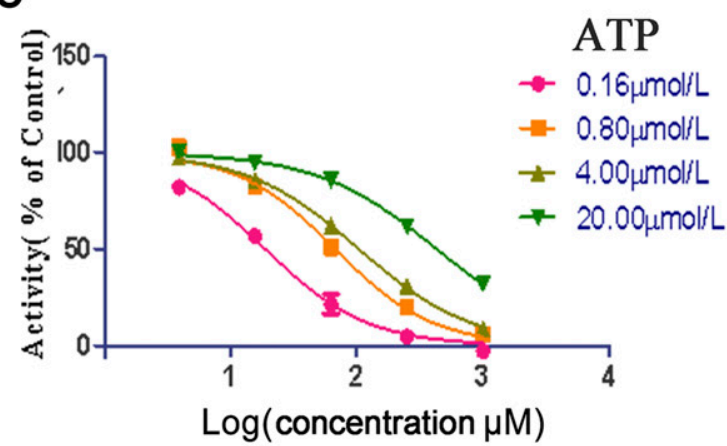

E
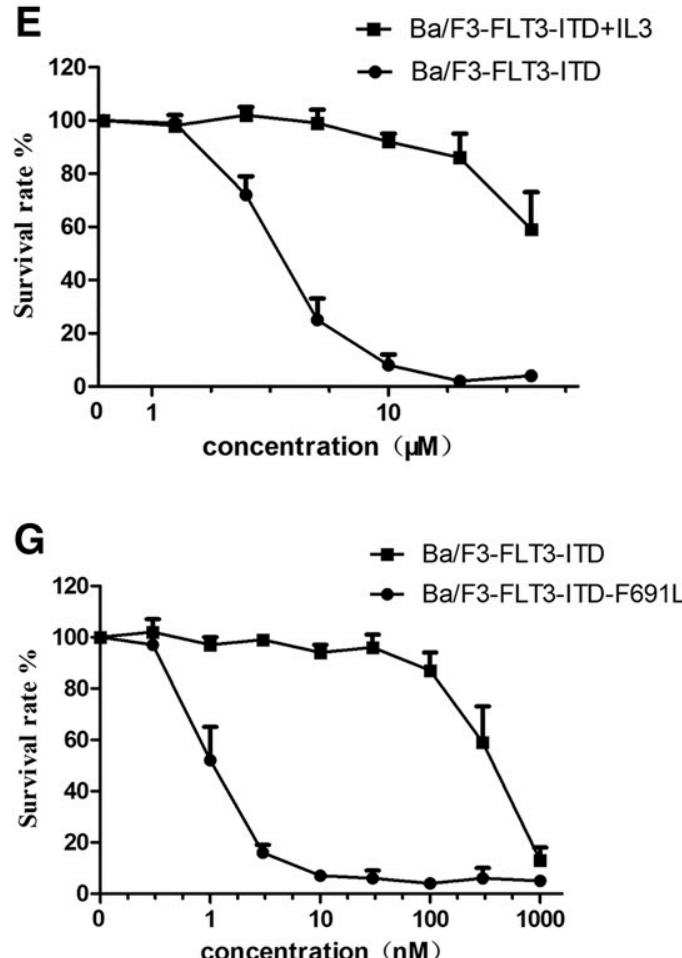

B

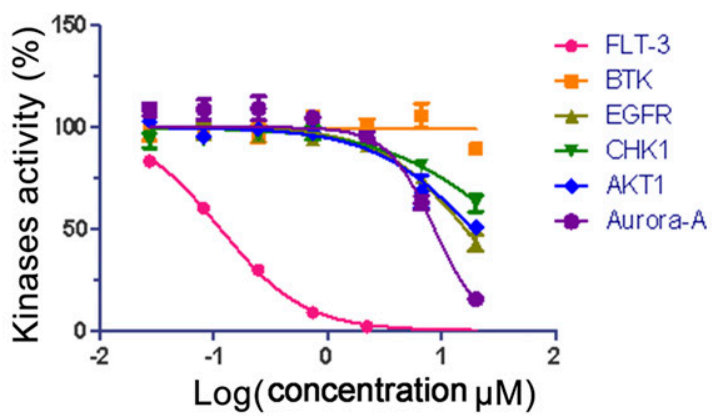

D

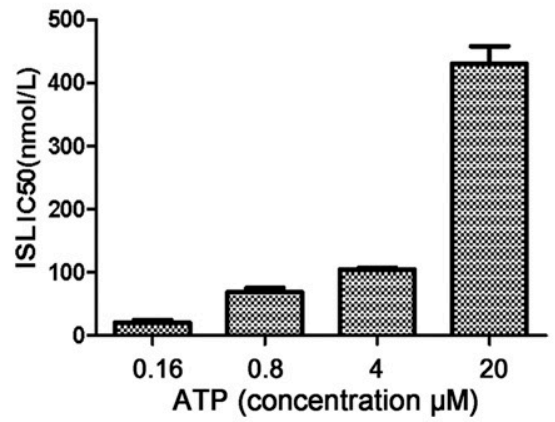

$\mathbf{F}$
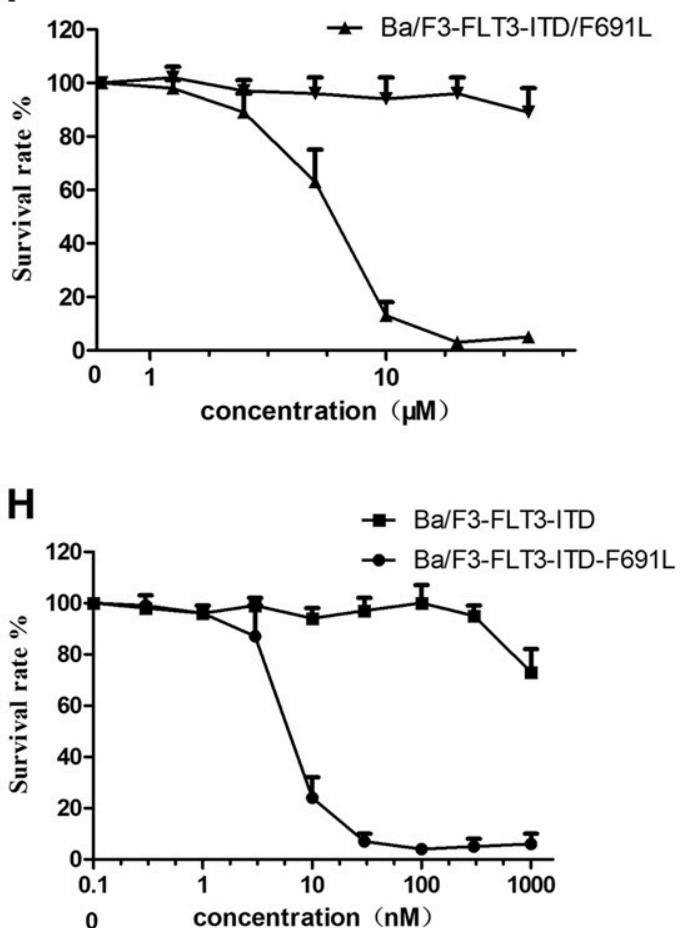

Fig. 1. ISL selectively suppressed FLT3 kinase activity and inhibited the proliferation of FLT3-dependent cells. (A) The chemical structure of ISL. (B) Kinase inhibitory activities of ISL were measured by the HTRF kit. ISL selectively inhibited FLT3 kinase activity. (C and D) The enzyme kinetic experiments were conducted with different concentrations of ATP. ISL is an ATP-competitive FLT3 inhibitor. (E) ISL selectively inhibited the proliferation of Ba/F3-FLT3-ITD cells and was nontoxic toward Ba/F3-FLT3-ITD cells rescued by IL-3. Ba/F3-FLT3-ITD cells cultured with or without IL3 were treated with ISL for 72 hours. Cell viability was measured by MTT assay. Data are representative of more than three independent experiments. (F) ISL selectively inhibited the proliferation of Ba/F3-FLT3-ITD/F691L cells and was nontoxic toward the cells rescued by IL-3. FLT3-ITD-F691L mutation is resistant to $\mathrm{AC} 220(\mathrm{G})$ and sorafenib $(\mathrm{H})$. 
Inhibitory Effect of ISL on FLT3 and Kinase Selectivity. In vitro, the enzyme kinetics assay revealed a remarkable inhibitory effect of ISL on FLT3 at the nanomolar concentration, with an $\mathrm{IC}_{50}$ value of $115.1 \pm 4.2 \mathrm{nM}$. The inhibitory effect of ISL on tyrosine kinase EGFR was noticeably weak, with an $\mathrm{IC}_{50}$ value of $16.19 \pm 2.4 \mu \mathrm{M}$. Conversely, ISL was largely inactive against tyrosine kinase $\mathrm{BTK}\left(\mathrm{IC}_{50}>\right.$ $20 \mu \mathrm{M})$. Meanwhile, ISL was highly selective for various types of Ser/Thr kinases, including CHK1, AKT1, and Aurora-A, with $\mathrm{IC}_{50}$ values of $>20 \mu \mathrm{M}, 19.0 \pm 3.8, \mu \mathrm{m}$ and $8.7 \pm 0.9 \mu \mathrm{m}$, respectively. These data suggested that ISL was a highly selective inhibitor of FLT3 (Fig. 1B). In addition, the enzyme kinetic experiments were conducted with a different concentration of ATP $(0.16-20 \mu \mathrm{M})$. With the increased concentration of ATP, the $\mathrm{IC}_{50}$ value of ISL to FLT3 also increased considerably from $20.0 \pm 7.3$ to $430.9 \pm 47.8 \mathrm{nM}$, demonstrating that ISL was an ATP-competitive FLT3 inhibitor (Fig. 1, C and D).

Selective Inhibition of Proliferation of Ba/F3-FLT3-ITD Cells by ISL. Next, ISL was tested for the ability to selectively
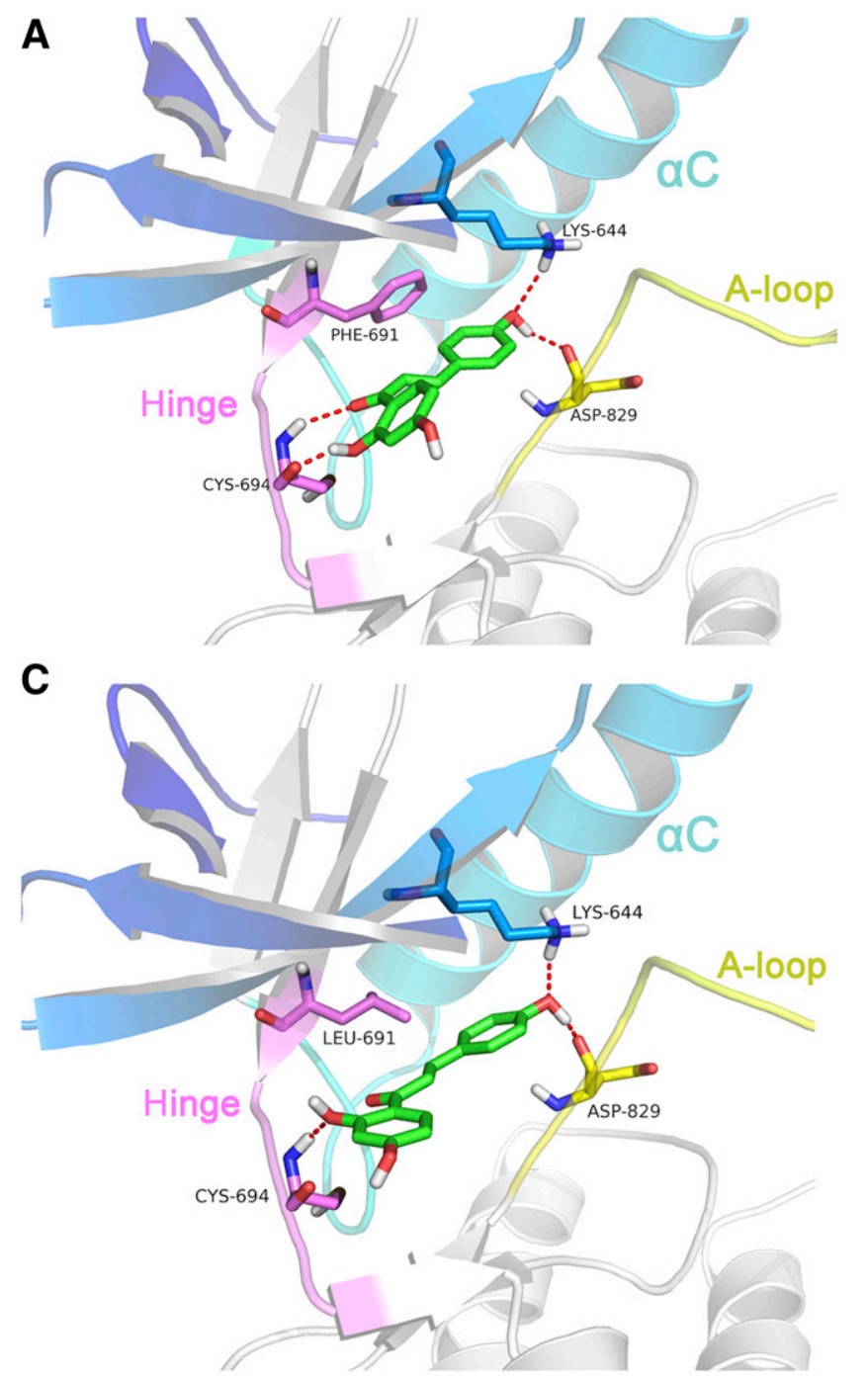

induce cell death in growth factor-independent Ba/F3-FLT3-ITD cells without affecting parental, growth factor [interleukin 3 (IL-3)]-dependent murine pro-B cell line Ba/F3. As shown in Fig. 1E, ISL significantly inhibited the proliferation of $\mathrm{Ba} /$ F3-FLT3-ITD cells at various concentrations between 2.5 and $40 \mu \mathrm{M}$, with an $\mathrm{IC}_{50}$ value of $2.9 \pm 0.8 \mu \mathrm{M}$ within $72 \mathrm{hours}$, and was nontoxic toward parental $\mathrm{Ba} / \mathrm{F} 3$ cells at concentrations up to $40 \mu \mathrm{M}$. However, Ba/F3-FLT3-ITD cells could be rescued from the antiproliferative effects of ISL by IL-3. These data suggested that ISL selectively inhibited the cell growth by targeting FLT3 and did not inhibit any other kinases in the signaling pathways used by the IL-3 receptor in $\mathrm{Ba} / \mathrm{F} 3$ cells.

FLT3-ITD/TKD (ITD plus F691L) dual mutation remains the main cause of the development of resistance to the FLT3 inhibitor. Among them, FLT3-ITD F691L mediated a noticeably strong resistance toward the FLT3 inhibitors AC220 and sorafenib. We performed proliferation studies of ISL on $\mathrm{Ba} / \mathrm{F} 3$ cells expressing FLT3-ITD/F691L and confirmed that ISL exhibited the ability to inhibit the proliferation of $\mathrm{Ba} / \mathrm{F} 3-\mathrm{FLT} 3-\mathrm{ITD} / \mathrm{F} 691 \mathrm{~L}$ with an $\mathrm{IC}_{50}$ value of $5.5 \pm 1.9 \mu \mathrm{M}$,

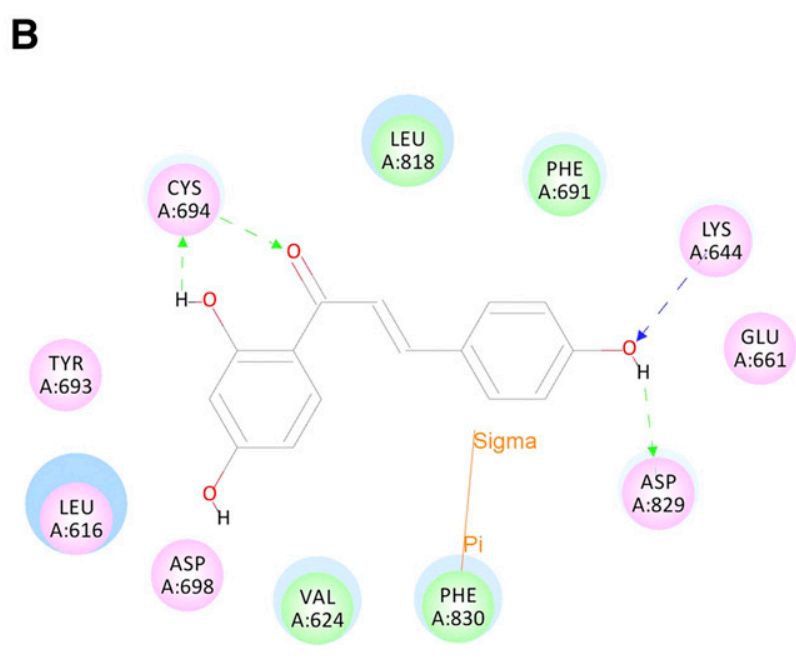

D

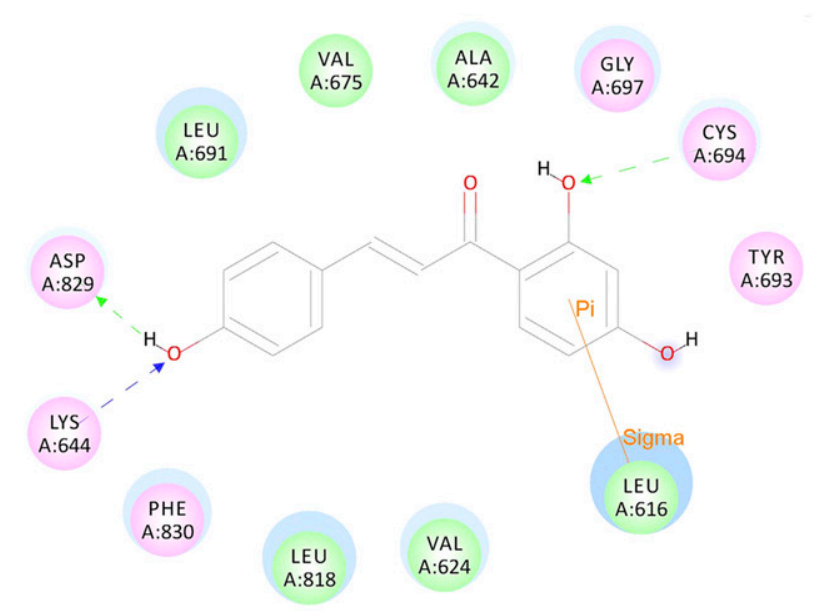

Fig. 2. The binding mode of ISL and FLT3. (A) ISL is docked into the FLT3-wt (wild type) kinase domain, demonstrating interactions between ISL and FLT3 (Protein Data Bank entry 4XUF). (B) The two-dimensional interaction map of ISL and FLT3-wt. (C) ISL is docked into the FLT3 kinase domain, showing interactions between ISL and FLT3 mutation (4XUF.M0005) in an inactive conformation. (D) The two-dimensional interaction map of ISL and FLT3/F691L. 
and $\mathrm{Ba} / \mathrm{F} 3-\mathrm{FLT} 3-\mathrm{ITD} / \mathrm{F} 691 \mathrm{~L}$ cells were also rescued by IL-3 (Fig. 1F). AC220 and sorafenib are very effective in inhibiting the proliferation of Ba/F3-FLT3-ITD cells, but they do not have an ideal effect on BA/F3-FLT3-ITD/F691L cells (Fig. 1, G and $\mathrm{H}$ ). These findings suggested that ISL could be used as a clinically active compound in patients with FLT3ITD mutation and patients with acquired FLT3-ITD/TKD dual mutation.

Predicted Binding Mode of ISL with FLT3. To investigate the possible binding mode of ISL with FLT3, the computational molecular docking studies were carried out using the DS 3.1 program package. The molecular docking studies were based on the known cocrystal structure of FLT3 with its inhibitor quizartinib (AC220; Protein Data Bank code 4XUF) (Smith et al., 2015; Zorn et al., 2015), and the binding mode of bioactive compound ISL with FLT3 is presented in Fig. 2. The carbonyl group and $4 \lambda^{3}$-benzene-1,3-diol group of ISL were hydrogens bonded with CYS694. In addition, the other two hydrogen bonds were also formed in the $4 \lambda^{3}$-phenol group with LYS644 and ASP829. Furthermore, one face-toface sigma- $\pi$ interaction was formed, which was between the hydrogen atom in the $4 \lambda^{3}$-phenol group and the benzene ring of PHE830. ISL also formed a hydrophobic interaction with residues LEU818, PHE691, PHE830, VAL624, and TYR693 in the ATP binding pocket of FLT3.

To illustrate the underlying mechanism of how ISL retains its activity against the F691L mutation, the protein models of the point mutation of FLT3/F691L were established. They were based on the cocrystal structure of FLT3 bound to AC220 (Protein Data Bank entry 4XUF) as a template by defining the "Build Mutants" module in the DS 3.1 program package. Five different protein models of point mutations were obtained after running the DS 3.1 program. The sequence was represented in tabular form according to their probability density function total energy from low to high (Table 2). Probability density function total energy of 4XUF.M0005 was recorded to be 1215.7684 , which was relatively lower than other energy values of the protein model. Thus, 4XUF.M0005 was selected as a receptor to further investigate the binding mode of ISL with the F691L mutation. From Fig. 2, it was revealed that the binding mode exhibited lower homology than the binding mode of ISL with FLT3; the stable combined conformation revealed that ISL could overcome the mutation of FLT3/F691L.

ISL Induces G0-G1 Phase Arrest and Apoptosis in AML Cells. Measurement of cellular DNA content and the analysis of the cell cycle were performed by flow cytometry. The cells were treated with different concentrations of ISL $(0.625,2.5$, and $10 \mu \mathrm{M})$ for 20 hours, and the levels of cell cycle progression were examined by flow cytometry. ISL

\section{TABLE 2}

The single mutated residue of FLT3 protein with the optimized local structure

\begin{tabular}{lccc}
\hline Name & PDF Total Energy $^{a}$ & PDF Physical Energy $^{a}$ & DOPE Score $^{a}$ \\
\hline 4XUF.M0005 & 1215.77 & 3.72 & $-33,513.02$ \\
4XUF.M0002 & 1215.78 & 3.75 & $-33,512.52$ \\
4XUF.M0003 & 1215.78 & 3.71 & $-33,512.18$ \\
4XUF.M0001 & 1215.78 & 3.76 & $-33,512.70$ \\
4XUF.M0004 & 1215.95 & 3.74 & $-33,511.29$ \\
\hline
\end{tabular}

DOPE, discrete optimized potential energy; PDF, probability density function ${ }^{a}$ Calculated by DS 3.1 (Accelrys, Inc.). significantly increased the number of MV4-11 cells in the G1 phase. The amount of cells in the G1 phase was increased from $52.52 \%$ to $66.70 \%$ (Fig. 3, A and C). In contrast, ISL markedly decreased the number of cells in the S phase and G2/ $\mathrm{M}$ in a dose-dependent manner, with the percentage decrease from $32.16 \%$ to $23.36 \%$ in S phase and $16.55 \%$ to $6.47 \%$ in G2/ $\mathrm{M}$, respectively. These results suggested that ISL could induce G0-G1 phase arrest in MV4- 11 cells.

ISL Induces Apoptotic Cell Death. Next, we investigated apoptotic cell death using annexin V-FITC assay. With the increasing concentration of ISL, the proportion of MV4-11 cells in apoptosis increased significantly from $3.92 \% \pm 2.65 \%$ to $53.11 \% \pm 11.31 \%$ (Fig. 3, B and D). For determination of the apoptotic pathway, we measured the expression of proteins involved in apoptosis by western blotting. The concentration of ISL increased $(2.5,10$, and $40 \mu \mathrm{M})$, and the cleavage of caspase-3 increased significantly, too (Fig. 4B). These results indicated that ISL could induce apoptosis in a dose-dependent manner at concentration above the $\mathrm{IC}_{50}$ value in $\mathrm{AML}$ cells.

Targeting of FLT3 Signaling Pathways by ISL in AML Cells. To determine the ability of ISL in blocking FLT3 and its downstream signaling, we screened some essential kinases involved in the FLT3 signaling pathway using western blot analysis. After a 20-hour treatment with gradient concentrations of ISL, MV4-11 cells were harvested and lysed for an immunoblot assay. As shown in Fig. 4A, ISL significantly suppressed the autophosphorylation of FLT3-ITD in a dose-dependent manner. Downstream signaling proteins Erk1/2 and STAT5 were also strongly inhibited by ISL at concentrations of $\geq 5 \mu \mathrm{M}$. Similarly, sunitinib (positive control) also inhibited the phosphorylation of FLT3, Erk1/2, and STAT5 in MV4-11 cells. Moreover, ISL did not interfere with the expression of these proteins during the treatment period. These findings suggested that ISL exerted its anti-AML function by directly targeting FLT3 displayed on the surface of leukemic cells and further antagonizing the FLT3-mediated downstream signaling cascade.

In Vivo Effects of ISL against MV4-11 Tumor Xenografts. The in vivo anti-AML activity of ISL was evaluated in the MV4-11 tumor xenograft model of NOD-SCID mice. The xenograft-bearing animals were treated orally with different doses of ISL at 100,50 , and $25 \mathrm{mg} / \mathrm{kg}$ per day or with vehicle alone. Oral sunitinib at a dose of $10 \mathrm{mg} / \mathrm{kg}$ per day was used as a positive control. As shown in Fig. 5A, treatment with ISL for 18 days exhibited a significant antitumor activity and inhibited MV4-11 tumor progression as compared with the vehicle group. Treatment with ISL at a dose of $100 \mathrm{mg} / \mathrm{kg}$ per day and treatment with sunitinib at $10 \mathrm{mg} / \mathrm{kg}$ per day elicited maximum tumor inhibition rates of $83.2 \%$ and $84.9 \%$, respectively, when compared with the vehicle-treated mice. ISL at doses of 50 and $25 \mathrm{mg} / \mathrm{kg}$ per day also significantly inhibited the MV4-11 tumor growth, with antitumor rates of $60.3 \%$ and $46.8 \%$, respectively. The body weight of mice was monitored once every 3 days during the course of the experiment. As illustrated in Fig. 5B, no significant loss in body weight was observed between the ISL-treated and vehicle-treated groups. No significant differences in gross measures, including skin ulcerations or toxic death, were observed in ISLtreated mice. Meanwhile, Yamazaki et al. (2002) proved that isoliquiritigenin (intraperitoneal injection) had an $\mathrm{LD}_{50}$ of about $3000 \mathrm{mg} / \mathrm{kg}$ in mice and did not cause animal death at 

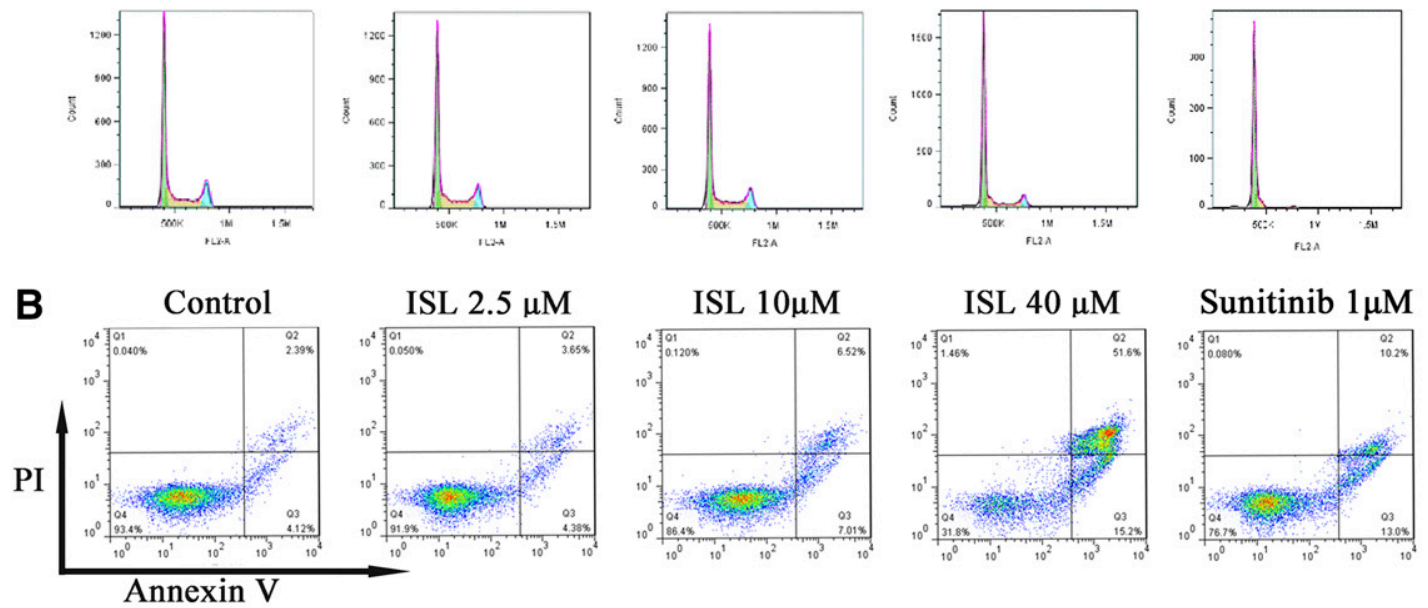

C

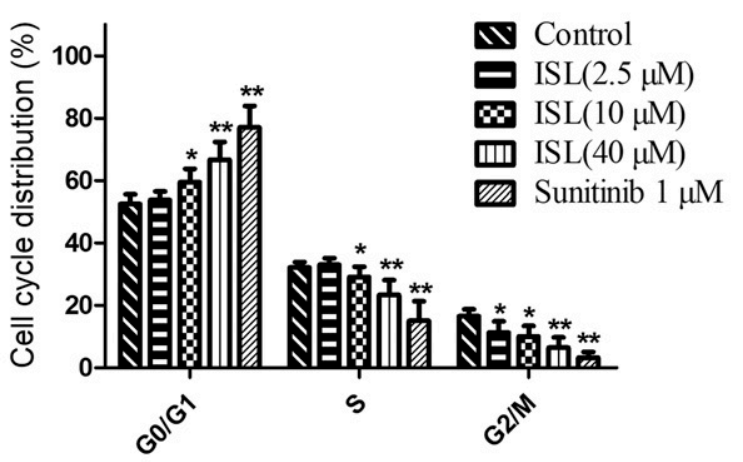

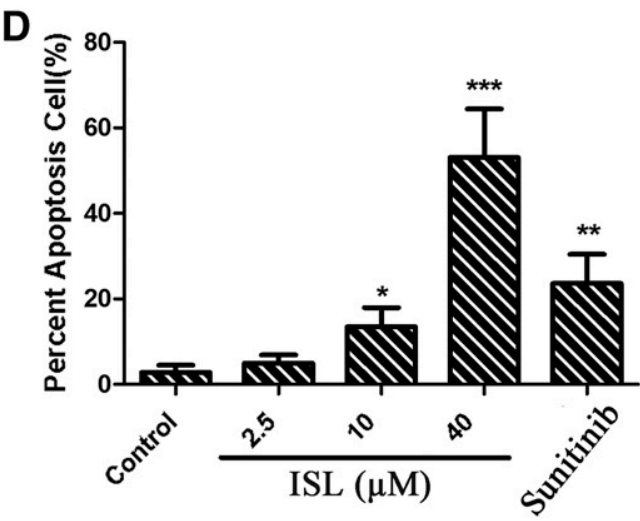

Fig. 3. ISL induced cell cycle arrest and apoptosis in AML cells. (A and C) MV4-11 cells were treated with serial concentrations of ISL for 20 hours. The cell cycle was measured by PI staining and flow cytometry. ISL significantly induced G0-G1 phase arrest with the decreased ratio of cells in the S to G2/M phase $(* P<0.05 ; * * P<0.01 ; n=3$ ). (B and D) MV4-11 cells were treated with ISL for 30 hours, and the apoptotic cells were defined by annexin-V and PI costaining. Apoptotic cells were defined as annexin-V+/PI- plus annexin-V+/PI+ cells. The ratio of apoptosis significantly increased from $3.92 \% \pm$ $2.65 \%$ (control) to $53.11 \% \pm 11.31 \%$ (ISL $40 \mu \mathrm{M} ; * P<0.05 ; * * P<0.01 ; * * * P<0.001 ; n=3$ ). Sunitinib, as a positive control, also significantly induced cell cycle arrest and apoptosis in AML cells.

$2000 \mathrm{mg} / \mathrm{kg}$. These data indicated that ISL inhibited the AML tumor growth in vivo without systemic toxicity.

In addition, the in vivo expression of p-Erk1/2 and Ki67 was measured by western blots and immunohistochemical analysis. As shown in Fig. 5D, the percentage of Ki67expressing and p-Erk1/2-positive cells in ISL-treated tumor tissue was significantly lower than in vehicle-treated tumor tissue. Meanwhile, the results of western blots also corroborated that ISL could inhibit the in vivo expression of p-Erk1/2 and Ki67 (Fig. 5E). The Ki67 protein expression was an established prognostic factor and was extensively used in the routine pathologic investigation as a proliferation marker or as a cell mitotic index. Furthermore, TUNEL assay was used to detect the in vivo cell apoptosis induced by ISL. As illustrated in Fig. 5D, tissue sections from the $100-\mathrm{mg} / \mathrm{kg}$ ISL-treated group possessed more TUNEL-positive cells, indicating a tumor with more apoptotic cells. These results demonstrated that ISL inhibited AML cell proliferation and induced apoptosis in vivo.

Evaluation of Survival in a Bone Marrow Model. To evaluate the influence of ISL on the survival time, we established a bone marrow engraftment model that mimics the pathology of human leukemia. MV4-11 cells were intravenously engrafted into mice after their endogenous bone marrow was ablated by pretreatment with cyclophosphamide. After allowing the disease to establish for 20 days, the mice were randomly divided into different groups (10 mice in each group), and treatment was initiated with 50 or $100 \mathrm{mg} / \mathrm{kg}$ per day ISL or with vehicle alone. Sunitinib at a dose of $10 \mathrm{mg} / \mathrm{kg}$ per day was used as a positive control. All vehicle group mice were presented with hind-limb paralysis or were moribund within 42-60 days, with a mean survival time (MST) of 52 days. The sunitinib-treated mice exhibited an MST of 70 days, and all animals expired by day 77. ISL-treated mice demonstrated prolonged survival in a dose-dependent manner; the MSTs were determined to be 60 and 68 days for the 50- and $100-\mathrm{mg} / \mathrm{kg}$ per day groups, respectively (Fig. 5C). In addition, we also determined the number and activity of MV4-11 cells in bone marrow. As shown in Fig. 5F, bone marrow MV4-11 cells in the vehicle group exhibited a strong nuclear immunoreactivity for Ki67, whereas other cells stained weakly. Treatment with ISL at $100 \mathrm{mg} / \mathrm{kg}$ per day resulted in reduced numbers of bone marrow MV4-11 cells when examined on day 40. In some cases of treatment with ISL, only a few leukemic cells distributed throughout the bone marrow remained. These findings suggested that ISL exhibited high efficacy in an FLT3-ITD model of lethal bone marrow disease in a dose-dependent manner. 
A

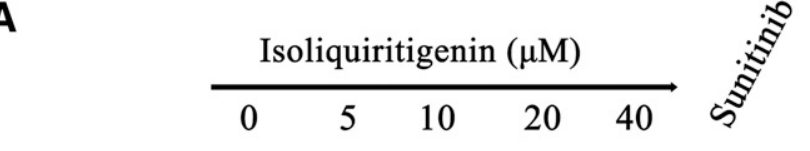

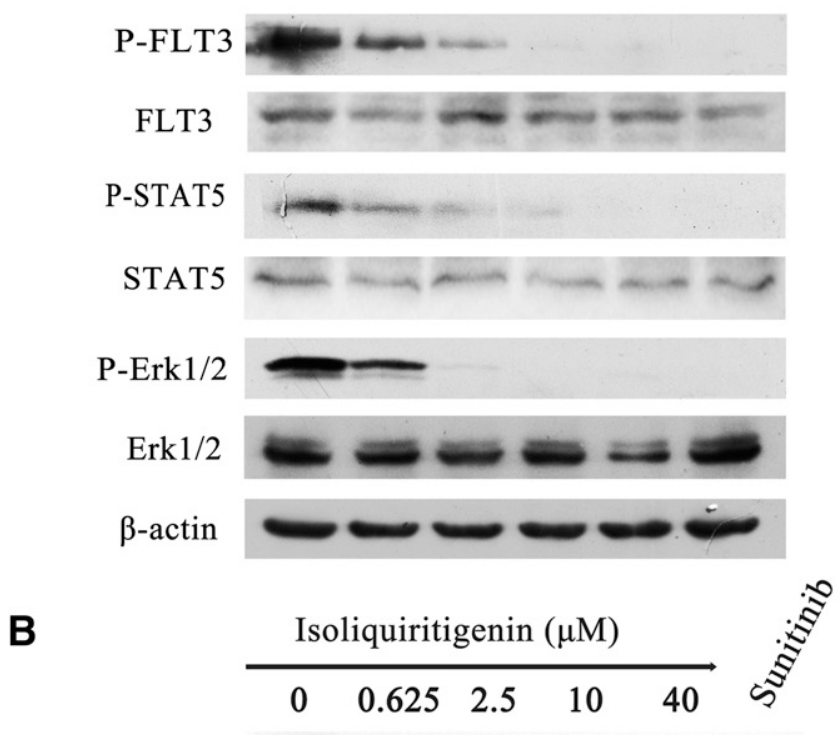

Caspase-3

Cleaved caspase-3

\section{GAPDH}

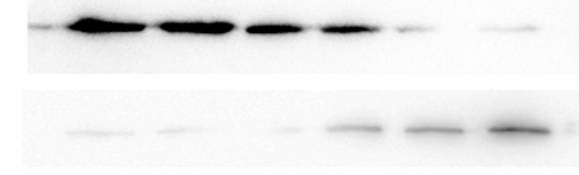

a

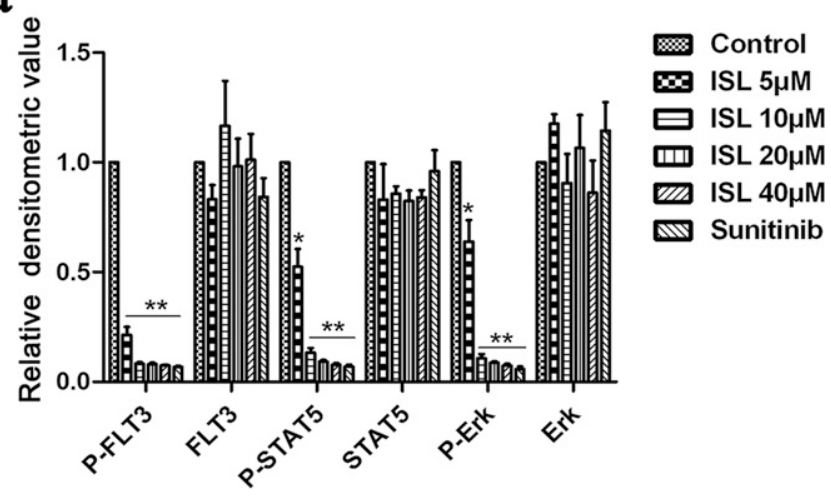

Fig. 4. ISL inhibited the activation of FLT3 signal and regulated the expressions of apoptosis-related protein. (A) After 16 hours of treatment with ISL, the phosphorylation of FLT3 and downstream signal proteins was assessed using western blot analysis. Sunitinib was considered as a positive control. (B) The cell lysate of MV4-11 cells treated with ISL for 30 hours was also analyzed using anti-caspase-3 antibody, and the results indicated a significant dose-dependent decrease in pro-caspase3 levels and a dose-dependent increase in cleaved caspase-3 levels with increasing concentrations of ISL. (a) The relative densitometric value of the western blot band was analyzed using image-analyzing software. Statistical analysis was performed to consider the differences $(* P<$ $0.05 ; * * P<0.01 ; n=3$ ). P-FLT3, phosphorylated FLT3; GAPDH, glyceraldehyde-3-phosphate dehydrogenase; P-STAT5, phosphorylated STAT5.

\section{Discussion}

AML is the most heterogeneous hematologic malignancy associated with a low survival rate in adults. Over the past few decades, tremendous technical advances in cytogenetic analysis have been made for early diagnosis and prognostic stratification. However, chemotherapy and hematopoietic stem cell transplantation remain the predominant treatment methods for AML, yet with a low 5-year survival rate (20\%) of AML patients (Schlenk et al., 2014; Ottmann et al., 2019). Related research has shown that the survival rate of patients using traditional Chinese medicine is considerably higher than that of traditional Chinese medicine nonusers (Huang et al., 2017). In particular, treatment with As2O3 is highly effective in AML patients with resistance to all-trans-retinoicacid and chemotherapy with relatively better safety (Chen et al, 1997; Wang and Chen, 2008; Li et al., 2017). Thus, traditional Chinese drugs may have potential therapeutic effects on AML.

Licorice, name derived from "gancao" in China, is a very well known herb in traditional Chinese medicine. It has been used for centuries and can be dated back to the Shen nong's Classic of Materia Medica around 2100 BC (Gibson, 1978). During the following millennia, licorice roots have been used for treating diseases such as influenza, coughs, and liver damage, and for detoxification (Kao et al., 2014). Recently, pharmacological studies have revealed numerous biologic activities associated with licorice, such as antiviral, antidiabetic, anti-inflammatory, antitumor, antimicrobial activities, and cholinergic regulation (Bode and Dong, 2015). Consequently, over 400 chemical components have been extracted and separated from licorice, containing nearly 300 flavonoids (Ji et al., 2016). However, the effective components in licorice responsible for these bioactivities remain elusive. Therefore, identification of their potential activity deserves more attention for development of a novel drug.

Isoliquiritigenin, a natural flavonoid constituent of licorice, possesses various medicinal bioactivities, including neuroprotective properties (Yang et al., 2016), analgesic properties (Shi et al., 2012), cardiac protection (Zhang et al., 2016), and anti-inflammatory properties (Zhang et al., $2018 b)$. There is a lot of evidence showing ISL has significant antitumor activity against many kinds of tumors, such as lung, colon, prostate, breast, gastric, and cervical cancer. It has also been shown to inhibit the VEGF/VEGFR-2 signal (Wang et al., 2013), suppress the activity of COX-2 (Zheng et al., 2014), increase CDK2 activity (Ii et al., 2004), and trigger reactive oxygen species in cancer cells (Kim et al., 2017). However, the anti-AML activities of ISL remain to be completely elucidated. Furthermore, potential targets of ISL are also largely unknown. Therefore, the present study aimed to investigate the anti-AML activity of ISL. We found that ISL could selectively inhibit FLT3-ITD mutant AML cells via targeting FLT3.

In vitro, ISL selectively inhibited the proliferation of FLT3-ITD mutant AML cell lines, including MV4-11 and MOLM-13, with $\mathrm{IC}_{50}$ values of $0.85 \pm 0.32$ and $1.27 \pm$ $0.53 \mu \mathrm{g} / \mathrm{ml}$, and exhibited weak toxicity effects or negligible activity in other tumor cell lines and normal cells. Moreover, ISL significantly inhibited the tumor growth of the MV4-11 xenograft model in vivo and significantly prolonged the survival time in the mouse bone marrow AML model 
A

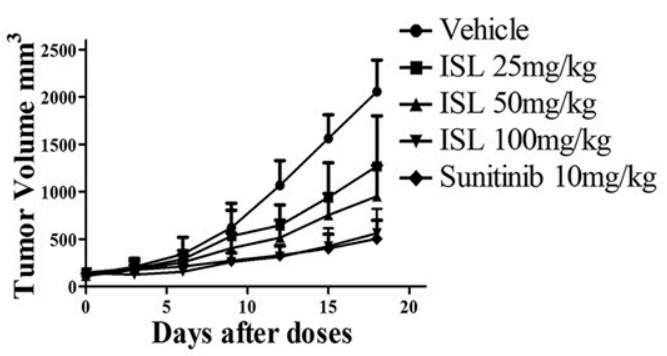

B

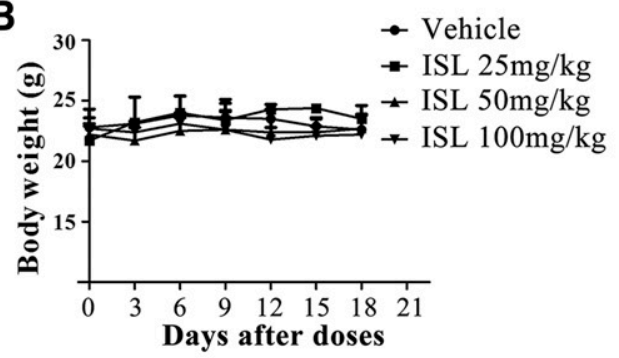

C

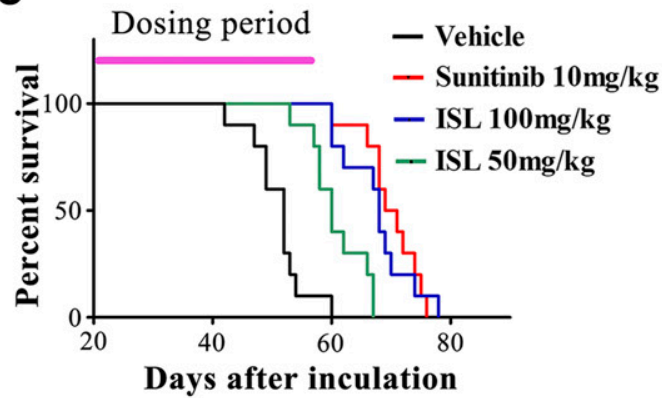

D

\section{Vehicle \\ ISL \\ $100 \mathrm{mg} / \mathrm{kg}$}
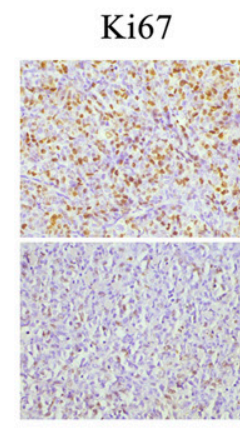

E

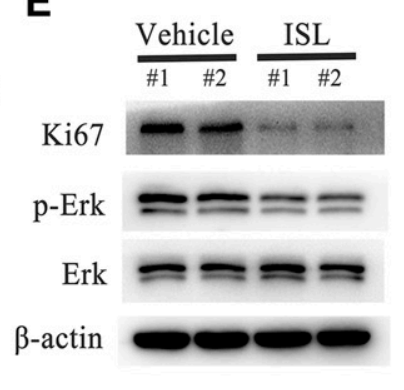

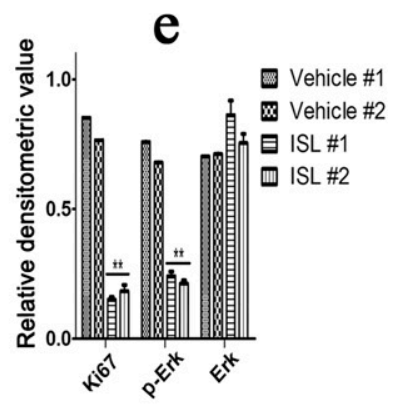

F

$\mathrm{Ki} 67$ in BM

Vehicle

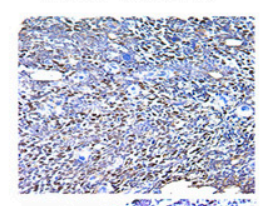

ISL

$100 \mathrm{mg} / \mathrm{kg}$

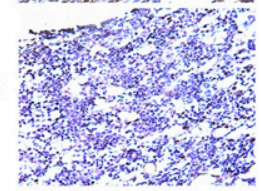

G

Heart

Liver

Spleen

Lung

Kidney

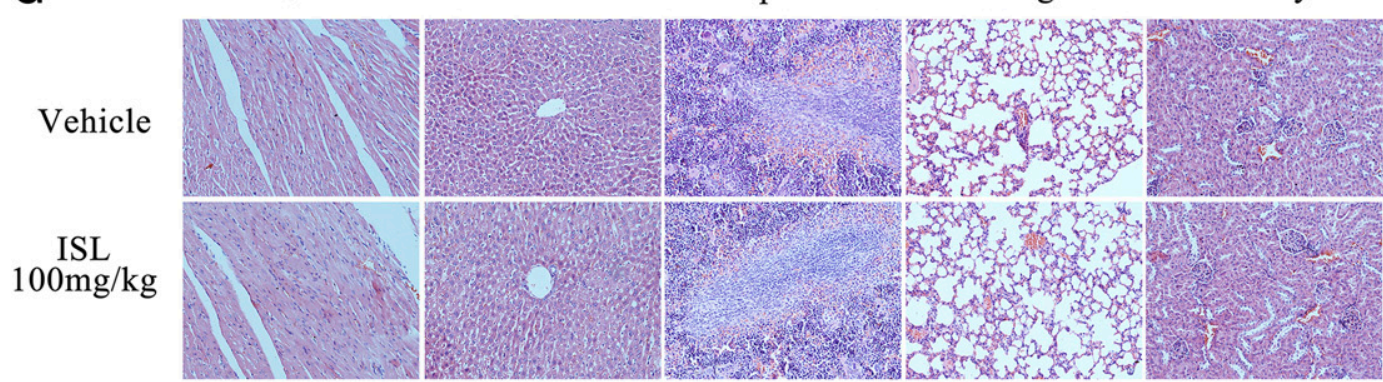

Fig. 5. In vivo effects of ISL against subcutaneous and bone marrow (BM) MV4-11 tumor models. (A) MV4-11 cells $\left(6 \times 10^{6} /\right.$ mouse) were subcutaneously injected into NOD-SCID mice, and oral treatment with ISL was initiated when the tumors grew to $100-200 \mathrm{~mm}^{3}$. ISL significantly inhibited the MV4-11 tumor growth at doses of $100(P<0.01 ; n=6), 50(P<0.01 ; n=6)$, or $25 \mathrm{mg} / \mathrm{kg}$ per day $(P<0.05 ; n=6)$ p.o. meanwhile, sunitinib as a positive control exhibited marked antitumor activity $(P<0.01 ; n=6)$. (B) The mouse body weights among the groups were analyzed after 18 days of treatment. The means \pm S.D. were also presented $(n=6)$. (C) Kaplan-Meier plot for survival analysis. NOD-SCID mice were intravenously injected with MV4-11 cells (5 $\times 10^{6}$ /mouse) and treated with an oral dose of ISL after 20 days of inoculation. Survival was determined by observation when the animals demonstrated hind-limb paralysis or became moribund. Finally, ISL significantly prolonged the survival time of bone marrow engraftment AML mice at doses of 100 $(P<0.001 ; n=10)$ and $50 \mathrm{mg} / \mathrm{kg}$ per day $(P<0.01 ; n=10)$ by mouth. (D) After 18 days of ISL treatment, MV4-11 tumors were harvested, fixed, and subjected to immunohistochemistry using Ki67 and p-Erk1/2 antibodies, followed by TUNEL assay. The findings of the study showed that ISL significantly inhibited the expression of Ki67, the activation of Erk1/2, and induced apoptosis in vivo. (E and e) MV4-11 tumors were also lysated for western blot analysis. The in vivo expression of Ki67 and activation of Erk1/2 was corroborated by western blot assay. The relative densitometric value (to the average value of the control group) of the western blot band was analyzed using image-analyzing software. Statistical analysis was performed to consider the differences $(P<0.05 ; * * P<0.01 ; n=3)$. (F) After 30 days of inoculation, bone marrow engraftment mice were treated with vehicle or ISL $(100 \mathrm{mg} / \mathrm{kg}$ per day) for 10 days. Subsequently, paraffin sections of bone marrow from the vehicle- or ISL-treated mice were stained with Ki67. The representative result is shown. (G) The analysis demonstrated that ISL had no influence on the micromorphology of normal tissues, including heart, liver, spleen, lung, and kidney. 
with better tolerance. Kinase profile data indicated that FLT3 was a selective target for ISL. Immunoblot assay revealed that ISL could effectively inhibit the phosphorylation of the FLT3/Erk1/2/STAT5 signaling pathway within 20 hours but did not regulate the expression of these proteins. Notably, ISL still retained a good inhibitory activity against FLT3-ITD/F691L mutation, a resistance kinase domain mutation against well known FLT3 inhibitors sorafenib and AC220. These results indicated that ISL could be a promising novel bioactive lead compound for AML treatment.

FLT3 is one of the most crucial drug targets in AML, because patients carrying FLT3-ITD mutations have poor response to standard chemotherapy, and the mutations inflict a poor prognosis in approximately $30 \%$ of AML cases (Gilliland and Griffin, 2002; Prada-Arismendy et al., 2017). FLT3 inhibitors, including midostaurin (Stone et al., 2017), quizartinib (AC220) (Cortes et al., 2018), ponatinib (Shah et al., 2013), and MLN-518 (Odia et al., 2016), have been demonstrated to inhibit the growth of FLT3-ITD-positive AML cells in animals and humans. Conversely, a clinical study proved that the FLT3-ITD-F691L mutation can cause acquired resistance to quizartinib (AC220) and other FLT3 inhibitors (Albers et al., 2013). Thus, we conducted proliferation studies of ISL in Ba/F3-FLT3-ITD/F691L cells and confirmed significant inhibitory activity of ISL on FLT3-ITD/F691L cells which is similar to that of FLT3-ITD cells. These cells could all be rescued by IL-3. Thus, ISL could also be expected to be clinically active in patients with acquired FLT3-ITD/TKD dual mutations.

In summary, isoliquiritigenin is an orally available natural bioactive compound, exhibiting anti-AML efficacy via targeting FLT3 both in vitro and in vivo. Therefore, this novel FLT3 inhibitor has the potential to contribute to the treatment of recurrence of FLT3-ITD mutant AML. In tandem with other anti-AML drugs, ISL might exhibit an enhanced therapeutic outcome in AML.

\section{Authorship Contributions}

Participated in research design: Cao, Wen, He, Chen, Peng.

Conducted experiments: Cao, Wen, He, Huang.

Contributed new reagents or analytic tools: Gao, Guo, Liu, Zheng, Gong, Li, Zhang.

Performed data analysis: Cao, Wen, He, Chen, Peng.

Wrote or contributed to the writing of the manuscript: Cao, Wen, He, Chen, Peng.

\section{References}

Albers C, Leischner H, Verbeek M, Yu C, Illert AL, Peschel C, von Bubnoff N, and Duyster J (2013) The secondary FLT3-ITD F691L mutation induces resistance to AC220 in FLT3-ITD+ AML but retains in vitro sensitivity to PKC412 and Sunitinib. Leukemia 27:1416-1418.

Bode AM and Dong Z (2015) Chemopreventive effects of licorice and its components. Curr Pharmacol Rep 1:60-71.

Boyapelly K, Bonin MA, Traboulsi H, Cloutier A, Phaneuf SC, Fortin D, Cantin AM, Richter MV, and Marsault E (2017) Synthesis and characterization of a phosphate prodrug of isoliquiritigenin. J Nat Prod 80:879-886.

Chen GQ, Shi XG, Tang W, Xiong SM, Zhu J, Cai X, Han ZG, Ni JH, Shi GY, Jia PM, et al. (1997) Use of arsenic trioxide (As2O3) in the treatment of acute promyelocytic leukemia (APL): I. As2O3 exerts dose-dependent dual effects on APL cells. Blood 89:3345-3353.

Cortes J, Perl AE, Döhner H, Kantarjian H, Martinelli G, Kovacsovics T, Rousselot P, Steffen B, Dombret H, Estey E, et al. (2018) Quizartinib, an FLT3 inhibitor, as monotherapy in patients with relapsed or refractory acute myeloid leukaemia: an open-label, multicentre, single-arm, phase 2 trial. Lancet Oncol 19:889-903.

Döhner H, Estey E, Grimwade D, Amadori S, Appelbaum FR, Büchner T, Dombret H, Ebert BL, Fenaux P, Larson RA, et al. (2017) Diagnosis and management of AML in adults: 2017 ELN recommendations from an international expert panel. Blood 129:424-447.

El Fakih R, Rasheed W, Hawsawi Y, Alsermani M, and Hassanein M (2018) Targeting FLT3 mutations in acute myeloid leukemia. Cells 7:E4.
Fiedler W, Kayser S, Kebenko M, Janning M, Krauter J, Schittenhelm M, Götze K, Weber D, Göhring G, Teleanu V, et al. (2015) A phase I/II study of sunitinib and intensive chemotherapy in patients over 60 years of age with acute myeloid leukaemia and activating FLT3 mutations. Br J Haematol 169:694-700.

Gibson MR (1978) Glycyrrhiza in old and new perspectives. Lloydia 41:348-354

Gilliland DG and Griffin JD (2002) The roles of FLT3 in hematopoiesis and leukemia. Blood 100:1532-1542.

Hsu YL, Kuo PL, and Lin CC (2005) Isoliquiritigenin induces apoptosis and cell cycle arrest through p53-dependent pathway in Hep G2 cells. Life Sci 77:279-292.

Huang L, Li H, Xie D, Shi T, and Wen C (2017) Personalizing Chinese medicine by integrating molecular features of diseases and herb ingredient information: application to acute myeloid leukemia. Oncotarget 8:43579-43591.

Ii T, Satomi Y, Katoh D, Shimada J, Baba M, Okuyama T, Nishino H, and Kitamura $\mathrm{N}$ (2004) Induction of cell cycle arrest and p21(CIP1/WAF1) expression in human lung cancer cells by isoliquiritigenin. Cancer Lett 207:27-35.

Ji S, Li Z, Song W, Wang Y, Liang W, Li K, Tang S, Wang Q, Qiao X, Zhou D, et al. (2016) Bioactive constituents of Glycyrrhiza uralensis (Licorice): discovery of the effective components of a traditional herbal medicine. J Nat Prod 79:281-292.

Kao TC, Wu CH, and Yen GC (2014) Bioactivity and potential health benefits of licorice. J Agric Food Chem 62:542-553.

Kim DH, Park JE, Chae IG, Park G, Lee S, and Chun KS (2017) Isoliquiritigenin inhibits the proliferation of human renal carcinoma Caki cells through the ROSmediated regulation of the Jak2/STAT3 pathway. Oncol Rep 38:575-583.

Kiyoi H, Ohno R, Ueda R, Saito H, and Naoe T (2002) Mechanism of constitutive activation of FLT3 with internal tandem duplication in the juxtamembrane domain. Oncogene 21:2555-2563.

Kundu P, Neese SL, Bandara S, Monaikul S, Helferich WG, Doerge DR, Khan IA, and Schantz SL (2018) The effects of the botanical estrogen, isoliquiritigenin on delayed spatial alternation. Neurotoxicol Teratol 66:55-62.

Levis MJ, Perl AE, Altman JK, Gocke CD, Bahceci E, Hill J, Liu C, Xie Z, Carson AR, McClain V, et al. (2018) A next-generation sequencing-based assay for minimal residual disease assessment in AML patients with FLT3-ITD mutations. Blood Adv 2:825-831.

Li YZ, Yu S, Yan PA, Gong DY, Wu FL, He Z, Yuan YY, Zhao AY, Tang X, Zhang RQ, et al (2017) Crotonoside exhibits selective post-inhibition effect in AML cells via inhibition of FLT3 and HDAC3/6. Oncotarget 8:103087-103099.

Liu B, Yang J, Wen Q, and Li Y (2008) Isoliquiritigenin, a flavonoid from licorice, relaxes Guinea-pig tracheal smooth muscle in vitro and in vivo: role of cGMP/PKG pathway. Eur J Pharmacol 587:257-266.

Odia Y, Sul J, Shih JH, Kreisl TN, Butman JA, Iwamoto FM, and Fine HA (2016) A Phase II trial of tandutinib (MLN 518) in combination with bevacizumab for patients with recurrent glioblastoma. CNS Oncol 5:59-67.

Ottmann OG, Müller-Tidow C, Krämer A, Schlenk RF, Lübbert M, Bug G, Krug U, Bochtler T, Voss F, Taube T, et al. (2019) Phase I dose-escalation trial investigating volasertib as monotherapy or in combination with cytarabine in patients with relapsed/refractory acute myeloid leukaemia. $\mathrm{Br}$ J Haematol 184:1018-1021.

Papaemmanuil E, Gerstung M, Bullinger L, Gaidzik VI, Paschka P, Roberts ND, Potter NE, Heuser M, Thol F, Bolli N, et al. (2016) Genomic classification and prognosis in acute myeloid leukemia. N Engl J Med 374:2209-2221.

Peng F, Du Q, Peng C, Wang N, Tang H, Xie X, Shen J, and Chen J (2015) A review: the pharmacology of isoliquiritigenin. Phytother Res 29:969-977.

Peng F, Tang H, Liu P, Shen J, Guan X, Xie X, Gao J, Xiong L, Jia L, Chen J, et al. (2017) Isoliquiritigenin modulates miR-374a/PTEN/Akt axis to suppress breast cancer tumorigenesis and metastasis. Sci Rep 7:9022.

Perl AE, Altman JK, Cortes J, Smith C, Litzow M, Baer MR, Claxton D, Erba HP, Gill S, Goldberg S, et al. (2017) Selective inhibition of FLT3 by gilteritinib in relapsed or refractory acute myeloid leukaemia: a multicentre, first-in-human, open-label, phase 1-2 study. Lancet Oncol 18:1061-1075.

Podoltsev NA, Stahl M, Zeidan AM, and Gore SD (2017) Selecting initial treatment of acute myeloid leukaemia in older adults. Blood Rev 31:43-62.

Prada-Arismendy J, Arroyave JC, and Röthlisberger S (2017) Molecular biomarkers in acute myeloid leukemia. Blood Rev 31:63-76.

Schlenk RF, Kayser S, Bullinger L, Kobbe G, Casper J, Ringhoffer M, Held G, Brossart P, Lübbert M, Salih HR, et al.; German-Austrian AML Study Group (2014) Differential impact of allelic ratio and insertion site in FLT3-ITD-positive AML with respect to allogeneic transplantation. Blood 124:3441-3449.

Shah NP, Talpaz M, Deininger MWN, Mauro MJ, Flinn IW, Bixby D, Lustgarten S, Gozgit JM, Clackson T, Turner CD, et al. (2013) Ponatinib in patients with refractory acute myeloid leukaemia: findings from a phase 1 study. $\mathrm{Br}$ J Haematol 162:548-552.

Shi Y, Wu D, Sun Z, Yang J, Chai H, Tang L, and Guo Y (2012) Analgesic and uterine relaxant effects of isoliquiritigenin, a flavone from Glycyrrhiza glabra. Phytother Res 26:1410-1417.

Siegel RL, Miller KD, and Jemal A (2015) Cancer statistics, 2015. CA Cancer J Clin 65:5-29.

Smith CC, Zhang C, Lin KC, Lasater EA, Zhang Y, Massi E, Damon LE, Pendleton M, Bashir A, Sebra R, et al. (2015) Characterizing and overriding the structural mechanism of the quizartinib-resistant FLT3 "gatekeeper" F691L mutation with PLX3397. Cancer Discov 5:668-679.

Stone RM, Mandrekar SJ, Sanford BL, Laumann K, Geyer S, Bloomfield CD, Thiede C, Prior TW, Döhner K, Marcucci G, et al. (2017) Midostaurin plus chemotherapy for acute myeloid leukemia with a FLT3 mutation. $N$ Engl J Med 377:454-464.

Tawata M, Aida K, Noguchi T, Ozaki Y, Kume S, Sasaki H, Chin M, and Onaya T (1992) Anti-platelet action of isoliquiritigenin, an aldose reductase inhibitor in licorice. Eur J Pharmacol 212:87-92.

Tsai CH, Hou HA, Tang JL, Liu CY, Lin CC, Chou WC, Tseng MH, Chiang YC, Kuo YY, Liu MC, et al. (2016) Genetic alterations and their clinical implications in older patients with acute myeloid leukemia. Leukemia 30:1485-1492.

Tsai JP, Lee CH, Ying TH, Lin CL, Lin CL, Hsueh JT, and Hsieh YH (2015) Licochalcone A induces autophagy through PI3K/Akt/mTOR inactivation and 
autophagy suppression enhances Licochalcone A-induced apoptosis of human cervical cancer cells. Oncotarget 6:28851-28866.

Wang N, Wang Z, Wang Y, Xie X, Shen J, Peng C, You J, Peng F, Tang H, Guan X et al. (2015) Dietary compound isoliquiritigenin prevents mammary carcinogenesis by inhibiting breast cancer stem cells through WIF1 demethylation. Oncotarget 6 9854-9876.

Wang Z, Wang N, Han S, Wang D, Mo S, Yu L, Huang H, Tsui K, Shen J, and Chen J (2013) Dietary compound isoliquiritigenin inhibits breast cancer neoangiogenesis via VEGF/VEGFR-2 signaling pathway. PLoS One 8:e68566.

Wang Z, Wang N, Liu P, Chen Q, Situ H, Xie T, Zhang J, Peng C, Lin Y, and Chen J (2014) MicroRNA-25 regulates chemoresistance-associated autophagy in breast cancer cells, a process modulated by the natural autophagy inducer isoliquiritigenin. Oncotarget 5:7013-7026.

Wang ZY and Chen Z (2008) Acute promyelocytic leukemia: from highly fatal to highly curable. Blood 111:2505-2515.

Wu M, Wu Y, Deng B, Li J, Cao H, Qu Y, Qian X, and Zhong G (2016) Isoliquiritigenin decreases the incidence of colitis-associated colorectal cancer by modulating the intestinal microbiota. Oncotarget 7:85318-85331.

Xu B, Zhao Y, Wang X, Gong P, and Ge W (2017) MZH29 is a novel potent inhibitor that overcomes drug resistance FLT3 mutations in acute myeloid leukemia. Leukemia 31:913-921.

Yamazaki S, Morita T, Endo H, Hamamoto T, Baba M, Joichi Y, Kaneko S, Okada Y, Okuyama T, Nishino H, et al. (2002) Isoliquiritigenin suppresses pulmonary metastasis of mouse renal cell carcinoma. Cancer Lett 183:23-30.

Yang EJ, Kim M, Woo JE, Lee T, Jung JW, and Song KS (2016) The comparison of neuroprotective effects of isoliquiritigenin and its Phase I metabolites against glutamate-induced HT22 cell death. Bioorg Med Chem Lett 26:5639-5643.

Yu SM and Kuo SC (1995) Vasorelaxant effect of isoliquiritigenin, a novel soluble guanylate cyclase activator, in rat aorta. $\mathrm{Br} J$ Pharmacol 114:1587-1594.
Zeng J, Chen Y, Ding R, Feng L, Fu Z, Yang S, Deng X, Xie Z, and Zheng S (2017) Isoliquiritigenin alleviates early brain injury after experimental intracerebral hemorrhage via suppressing ROS- and/or NF-кB-mediated NLRP3 inflammasome activation by promoting Nrf2 antioxidant pathway. J Neuroinflammation 14:119.

Zhang B, Lai Y, Li Y, Shu N, Wang Z, Wang Y, Li Y, and Chen Z (2018a) Antineoplastic activity of isoliquiritigenin, a chalcone compound, in androgen-independent human prostate cancer cells linked to G2/M cell cycle arrest and cell apoptosis. Eur J Pharmacol 821:57-67.

Zhang W, Tao Q, Guo Z, Fu Y, Chen X, Shar PA, Shahen M, Zhu J, Xue J, Bai Y, et al. (2016) Systems pharmacology dissection of the integrated treatment for cardiovascular and gastrointestinal disorders by traditional Chinese medicine. Sci Rep 6 32400 .

Zhang W, Wang G, and Zhou S (2018b) Protective effects of isoliquiritigenin on LPSinduced acute lung injury by activating PPAR- $\gamma$. Inflammation 41:1290-1296.

Zheng H, Li Y, Wang Y, Zhao H, Zhang J, Chai H, Tang T, Yue J, Guo AM, and Yang $\mathrm{J}$ (2014) Downregulation of COX-2 and CYP 4A signaling by isoliquiritigenin inhibits human breast cancer metastasis through preventing anoikis resistance, migration and invasion. Toxicol Appl Pharmacol 280:10-20.

Zorn JA, Wang Q, Fujimura E, Barros T, and Kuriyan J (2015) Crystal structure of the FLT3 kinase domain bound to the inhibitor quizartinib (AC220). PLoS One 10: e0121177.

Address correspondence to: Jian-Ping Chen, School of Chinese medicine, University of Hong Kong, Pokfulam 999077, Hong Kong, China. E-mail: abchen@hku.hk; or Cheng Peng, Pharmacy College, Chengdu University of Traditional Chinese Medicine, No. 1166, Liutai Road, Wenjiang District, Chengdu 611137, China. E-mail: pengchengchengdu@126.com 